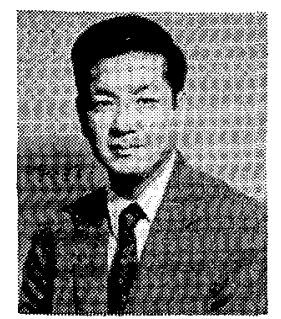

Hing C. So (M'60-SM'74) received the B.S., M.S., and $P h . D$. degrees in electrical engineering from the University of Illinois, Urbana, in 1956, 1957 , and 1960, respectively.

From 1958 to 1960 he was an Instructor in electrical engineering at the University of Illinois. In 1960 he joined the faculty of the Department of Electrical Engineering, University of Rochester, Rochester, NY, where he was appointed Associate Professor in 1965. During the academic year 1963-1964 he was on leave from the University of Rochester and was at Bell Laboratories, Murray
Hill, NJ. He left Rochester to join Bell Laboratories in September 1965, where he worked on computer graphics, user-oriented computer languages, system theory, and computer-aided circuit analysis and design. Since 1971 he has been at Bell Laboratories, Whippany, NJ, where he has been engaged in research in computer algorithms for the interconnection of large digital systems. He has lectured at Columbia University, New York as Adjunct Professor of electrical engineering and computer science. $\mathrm{He}$ is a former Associate Editor of the IEEE TRANSACTIONS ON Circuirs AND SYstems.

Dr. So is a member of the Technical Committee on Large Scale Systems of the IEEE Circuits and Systems Society. He is a member of Tau Beta Pi, Eta Kappa Nu, and Sigma Xi.

\title{
A New Algorithm for Solution of Resistive Networks Involving Diodes
}

\author{
DIMITRI P. BERTSEKAS
}

\begin{abstract}
The solution of electric network problems by various algorithms such as for example Newton's method is often hampered by the presence of physical diodes with steeply rising exponential characteristics which cause overflow and slow convergence during numerical computation. In this paper we propose and analyze an algorithm which bypasses these difficulties by successively approximating the steep diode characteristics by smoother exponential functions. The algorithm may be modified to be used in the presence of ideal diodes and is related to penalty and multiplier methods for constrained minimization and Davidenko's method for solving certain ill-conditioned systems of nonlinear equations.
\end{abstract}

\section{INTRODUCTION}

C ONSIDER a connected nonlinear resistive electric network consisting of $m$ nodes and $r$ arcs. Let $i_{n}$ denote the current through the $n$th arc and $v_{n}$ denote the voltage difference across the $n$th arc. Let $i=\left(i_{1}, \cdots, i_{r}\right)^{\prime}$ and $v=\left(v_{1}, \cdots, v_{r}\right)^{\prime}$ denote the corresponding $r$-dimensional (column) vectors. If $A$ is the incidence matrix of the network and $B$ is the loop matrix of the network we have

Manuscript received October 24, 1975; revised June 11, 1976. This work was supported by the National Science Foundation under Grant ENG 74-19332.

The author is with the Department of Electrical Engineering and the Coordinated Science Laboratory, University of Illinois, Urbana, IL 61801 . from Kirchhoff's current and voltage laws

$$
\begin{aligned}
A i & =0 \\
B v & =0 .
\end{aligned}
$$

As is well known (see e.g., [1]), the equations above yield a set of $r$ independent equations in $(i, v)$. Suppose that $s$, with $1 \leqslant s<r$, out of the $r$ arcs of the network consist of physical diodes with exponential characteristics of the form

$$
g_{n}\left(i_{n}, v_{n}\right)=i_{n}-p_{n}\left(e^{q_{n} v_{n}}-1\right)=0, \quad n=1, \cdots, s
$$

where $p_{n}$ and $q_{n}$ are given positive scalars, while there are $(r-s)$ additional equations relating $i$ and $v$ of the form

$$
f_{n}(i, v)=0, \quad n=s+1, \cdots, r
$$

where $f_{n}$ are continuously differentiable functions.

The numerical solution of the system of equations (1), (2), (3) can be attempted by application of Newton's method. However this approach in practice often meets with formidable difficulties when the coefficients $q_{n}$ in the diode characteristic equations (2) are large (a typical range of values for transistor circuits is $q_{n}=30-40$ ). First, as predicted by Kantorovich's theorem (see e.g., [2]), the region around a solution where Newton's method exhibits 
fast convergence depends strongly on the magnitude of the second derivatives of the functions $g_{n}$ and $f_{n}$. When $q_{n}$ is large the second derivative of $g_{n}$ with respect to $v_{n}$ is very large even for moderate positive values of $v_{n}$. As a result Newton's method can converge very slowly unless the starting point is very near a solution. Second, when $q_{n}$ is large the range of values $e^{q_{n} v_{n}}$ can take is very large with overflow easily resulting during numerical computation.

The numerical difficulties associated with straightforward application of Newton's method in diode network problems are widely recognized (see e.g., Calahan [3]) and a number of approaches [4]-[7], [23] have been suggested in the literature for overcoming these difficulties. One of the approaches suggested has been the employment in a particular way of Davidenko's method [8] for solving ill-conditioned systems of nonlinear equations [9]. In this paper we propose an algorithmic approach similar to Davidenko's but yet different in some important aspects which results, in our opinion, in considerably more efficient computation. Our approach is based on an equivalence between network problems and optimization problems which has been known for some time and has been sharpened to a definitive form recently by Minty [10], Rockafellar [11], [12], and others in the context of modern duality theory. It is also based on some recent ideas in the theory of computational optimization which have given rise to a class of methods for constrained optimization called methods of multipliers (see [13] for a literature survey). This class of methods has several important advantages over the class of penalty function methods and tends to replace them in most applications. The algorithm considered in this paper is directly related to a method of multipliers with exponential penalty function first proposed by B. Kort and the author in 1972 [14]. In the next section we describe our algorithm while in Section III we present results of computational experiments. In Section IV we explain the motivation of the algorithm and we provide a convergence result for the case of a monotone network. From the discussion of Section IV it will become cvident that similar algorithms may be constructed to treat other types of "ill-conditioned" arc characteristics involving, for example, nondifferentiabilities, discontinuities, etc. Examples of this type of algorithms have been provided in separate publications [15], [16], [24], [25].

\section{The Algorithm}

Consider the possibility of replacing (2) by equations of the form

$$
\tilde{g}_{n}\left(i_{n}, v_{n}, y_{n}, \epsilon\right)=0, \quad n=1, \cdots, s
$$

where $y_{n}>0$ and $\epsilon \geqslant 0$ are scalar parameters and $\tilde{g}_{n}$ is defined by

$$
\begin{aligned}
& \tilde{g}_{n}\left(i_{n}, v_{n}, y_{n}, \epsilon\right)=i_{n} \\
& \quad-\left(y_{n}\right)^{q_{n} \epsilon /\left(1+q_{n} \epsilon\right)}\left(p_{n}\right)^{1 /\left(1+q_{n} \epsilon\right)} e^{q_{n} v_{n} /\left(1+q_{n} \epsilon\right)}+p_{n} .
\end{aligned}
$$

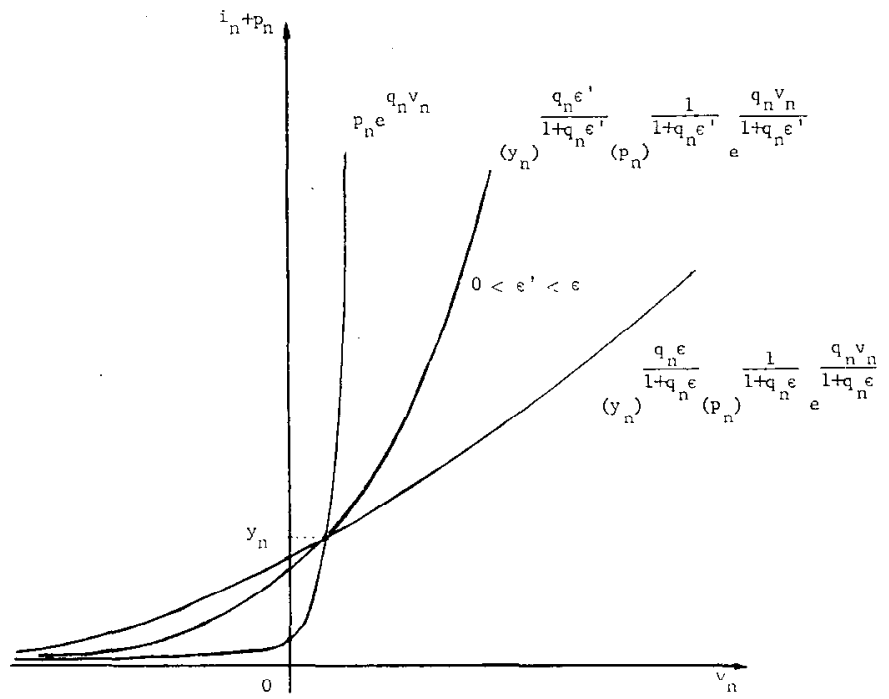

Fig. 1.

The functions $g_{n}$ and $\tilde{g}_{n}$ are depicted in Fig. 1. Notice that $\lim _{\epsilon \rightarrow 0^{+}} \tilde{g}_{n}\left(i_{n}, v_{n}, y_{n}, \epsilon\right)=g_{n}\left(i_{n}, v_{n}\right)$

$$
\forall i_{n}, v_{n} \in R, \quad y_{n}>0, \quad n=1, \cdots, s .
$$

In fact it is easy to see that if $\left\{i_{n}^{k}\right\},\left\{v_{n}^{k}\right\}$ are convergent sequences with $i_{n}^{k} \rightarrow \bar{i}_{n}, v_{n}^{k} \rightarrow \bar{v}_{n},\left\{y_{n}^{k}\right\}$ is any sequence of positive numbers bounded above and bounded away from zero, $\left\{\epsilon_{k}\right\}$ is a sequence of nonnegative numbers and furthermore

$$
\epsilon_{k} \rightarrow 0, \quad \tilde{g}_{n}\left(i_{n}^{k}, v_{n}^{k}, y_{n}^{k}, \epsilon_{k}\right) \rightarrow 0
$$

then we must have

$$
g_{n}\left(\vec{i}_{n}, \bar{v}_{n}\right)=0
$$

Based on the observations above, a possible approach for solving the system of equations (1)-(3) is to specify sequences $\left\{y_{n}^{k}\right\}, n=1, \cdots, s,\left\{\epsilon_{k}\right\}$, and $\left\{\gamma_{k}\right\}$ with

$$
\begin{array}{lll}
0<\delta \leqslant y_{n}^{k} \leqslant \Delta, & \forall k, & \text { for some } \delta, \Delta \\
0 \leqslant \epsilon_{k+1} \leqslant \epsilon_{k}, & \forall k, & \epsilon_{k} \rightarrow 0 \\
0 \leqslant \gamma_{k+1} \leqslant \gamma_{k}, & \forall k, & \gamma_{k} \rightarrow 0
\end{array}
$$

and find for each index $k$ vectors $\left(i^{k}, v^{k}\right)$ which approximately solve the system of equations (1), (3), (4) in the sense that

$$
\begin{gathered}
\left|\tilde{g}_{n}\left(i_{n}^{k}, v_{n}^{k}, y_{n}^{k}, \epsilon_{k}\right)\right| \leqslant \gamma_{k}, \quad n=1, \cdots, s \\
\left|f_{n}\left(i^{k}, v^{k}\right)\right| \leqslant \gamma_{k}, \quad n=s+1, \cdots, r \\
\left\|A i^{k}\right\| \leqslant \gamma_{k} \\
\left\|B v^{k}\right\| \leqslant \gamma_{k}
\end{gathered}
$$

where $\|\cdot\|$ denotes the usual Euclidean norm. Then any limit points $\bar{i}, \bar{v}$ of the sequences $\left\{i^{k}\right\},\left\{v^{k}\right\}$ constitute a solution of the network equations (1)-(3). In other words successive approximate solution (within $\gamma_{k}$ ) of the system 
of equations (1), (3), (4) yields in the limit solutions of the system of equations (1)-(3).

Notice that finding $\left(i^{k}, v^{k}\right)$ satisfying (10)-(12) by means of Newton's method will not present difficulties for moderately large values of $\epsilon_{k}$ since now the exponential in (5) rises much less steeply than in the diode equation (2). Thus by starting with relatively large values of $\epsilon($ say $\epsilon=1)$ and progressively reducing $\epsilon$ towards zero one gradually approaches a solution of the problem in a manner which alleviates the effects of "ill-conditioning" much in the spirit of Davidenko's method and penalty function methods in constrained minimization.

An important additional feature of the method proposed is the introduction of the scalar parameters $y_{n}^{k}$, $n=1, \cdots, s$. While the algorithm would work if the scalars $y_{n}^{k}$ were held constant (say fixed at the value $y_{n}^{k}=1$ for all $k$ ) we propose to update $y_{n}^{k}$ according to the equation

$$
\begin{array}{r}
y_{n}^{k+1}=\left(y_{n}^{k}\right)^{q_{n} \varepsilon_{k} /\left(1+q_{n} \varepsilon_{k}\right)}\left(p_{n}\right)^{1 /\left(1+q_{n} \varepsilon_{k}\right)} e^{q_{n} v_{n}^{k} /\left(1+q_{n} \epsilon_{k}\right),} \\
n=1, \cdots, s .
\end{array}
$$

Notice that from (5), (10), and (13) we have for all $k$

$$
\left|i_{n}^{k}+p_{n}-y_{n}^{k+1}\right| \leqslant \gamma_{k}, \quad n=1, \cdots, s .
$$

As a result if the sequence $\left\{v_{n}^{k}\right\}$ generated by the algorithm is bounded we have (using (5), (10)) that the sequence $\left\{i_{n}^{k}+p_{n}\right\}$ after some sufficiently large index is positive and bounded away from zero. It follows, in view of (14) and the fact $\gamma_{k} \rightarrow 0$, that the sequence $\left\{y_{n}^{k+1}\right\}$ is bounded away from zero for every $n=1, \cdots, s$ as required by (7). It turns out that in many cases the algorithm proposed is much more efficient when the updating formula (13) is utilized than when the parameters $y_{n}^{k}$ are left constant. In fact the algorithm may work satisfactorily even if the parameter $\epsilon_{k}$ is not decreased to zero but rather is held constant at some positive value. In other words convergence may be induced by iteration (13) alone. This fact will be proved for the case of monotone networks in Section IV. For other cases there are no analytical results presently available. However experimental evidence supports the conjecture that the employment of (13) will accelerate convergence in the great majority of cases of interest.

It is worth noting that, with minor modifications, the algorithm can be also applied to problems with ideal rather than physical diodes, i.e., for diodes with characteristic equation

$$
i_{n}=\left\{\begin{array}{ll}
0, & \text { if } v_{n} \leqslant 0 \\
+\infty, & \text { if } v_{n}>0
\end{array} .\right.
$$

The approximating function $\tilde{g}_{n}$ in this case takes the form

$$
\tilde{g}_{n}\left(i_{n}, v_{n}, y_{n}, \epsilon\right)=i_{n}-y_{n} e^{v_{n} / \epsilon}=0
$$

and the multiplier iteration (13) takes the form

$$
y_{n}^{k+1}=y_{n}^{k} e^{v_{n}^{k} / \epsilon_{k}}, \quad n=1, \cdots, s .
$$

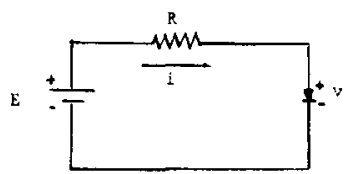

Fig. 2.

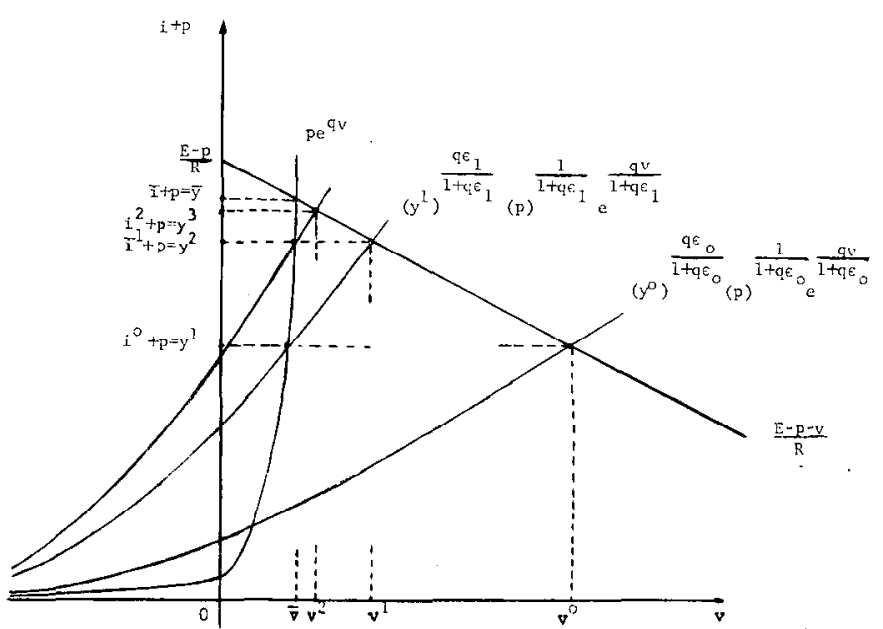

Fig. 3.

We note also that it is possible to use an approximating function for an ideal diode which is not exponential. Such functions may be obtained from corresponding members of a general class of penalty functions introduced in [17]-[19] in the manner explained in Section IV.

We end this section by demonstrating pictorially how the algorithm will work for the case of a simple example network.

\section{Example}

Consider the network shown in Fig. 2 where the diode shown is either ideal or has an exponential characteristic. We assume that the solution of the approximating network is exact, i.e., $\gamma_{k} \equiv 0$.

Let $i$ and $v$ denote the current and voltage difference across the diode and let them be related by

$$
i=p\left(e^{q v}-1\right) \text {. }
$$

The successive iterations of our algorithm (with $\gamma_{k} \equiv 0$ ) are shown in Fig. 3. Notice the mechanism by which the multipliers $y^{k}$ are generated and the fact that the "approximating" exponential characteristic

$$
\left(y^{k}\right)^{q \epsilon_{k} /\left(1+q \epsilon_{k}\right)}(p)^{1 /\left(1+q \epsilon_{k}\right)} e^{q v /\left(1+q \epsilon_{k}\right)}
$$

intersects the diode exponential $p e^{q v}$ at the point with vertical coordinate $y^{k}=i^{k-1}+p$. It is easy to see in the example above that convergence to the pair $(\bar{i}, \bar{v})$ can be induced by the multiplier iteration (13) alone without the need to decrease $\epsilon_{k}$ to zero thus completely eliminating the ill-conditioning problem. It is to be noted however that 


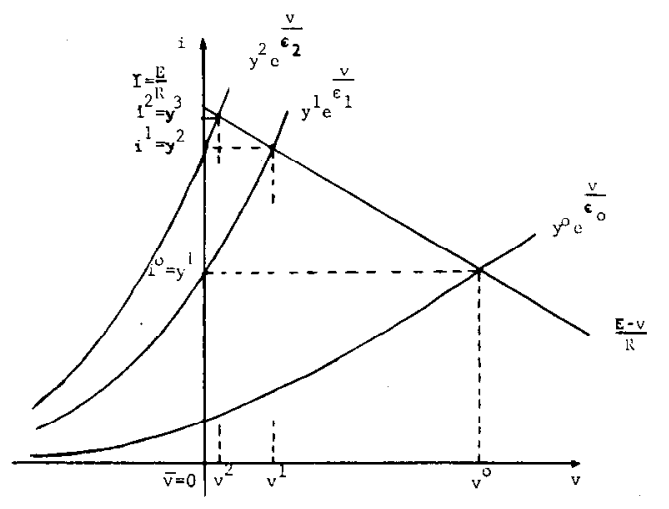

Fig. 4.

convergence will be faster when $\epsilon_{k}$ is successively reduced to zero rather than kept constant.

Consider now the case where the diode in Fig. 2 is an ideal diode. Then the solution of the problem is trivially $\bar{i}=E / R, \bar{v}=0$. Fig. 4 shows how our algorithm will find this solution.

\section{Computational Aspects and Results}

The algorithm presented in this paper has been utilized to solve a few test problems. Generally the method performed in a reliable manner. In our experimental program the pure form of Newton's method for solving nonlinear equations (i.e., with stepsize equal to one) was utilized throughout. In a general purpose program however one would certainly need to modify Newton's method by including a steplength procedure or other devices which would improve reliability. The algorithmic solution of each "approximate" network problem was terminated when the absolute value of each of the equations was less than $10^{-5}\left(\gamma_{k} \equiv 10^{-5}\right.$ in (10)-(12)).

A special automatic procedure was utilized to determine the sequence of parameters $\left\{\epsilon_{k}\right\}$. At the beginning of the computation a sufficiently large initial value $\epsilon_{0}$ was determined such that the values of all equations at the initial point $\left(i^{0}, v^{0}, y^{0}\right)$ were reasonable (say $\left.<10^{3}\right)$. At the beginning of the $k$ th solution cycle a trial value $\epsilon_{k}=$ $\epsilon_{k-1} / 8$ was adopted and this value was decreased or increased depending on whether the values of all equations at the point $\left(i^{k-1}, v^{k-1}, y^{k}\right)$ was within certain ranges. (In our program we decreased $\epsilon$ by a factor of 8 if all initial equation values were less than $10^{-3}$ and we increased $\epsilon$ by a factor of 2 if some initial equation values were greater than unity. We took however always $\epsilon_{k} \leqslant$ $0.5 \epsilon_{k-1}$ so that the sequence $\left\{\epsilon_{k}\right\}$ was always monotonically decreasing.) This automatic procedure for adjusting the approximation parameter $\epsilon$ is aimed at striking a reasonable balance between fast convergence of the algorithm and reduction of ill-conditioning.

From computational experience as wcll as theoretical considerations based on general properties of multiplier methods (see, e.g., [13]) the rate of convergence is accel-

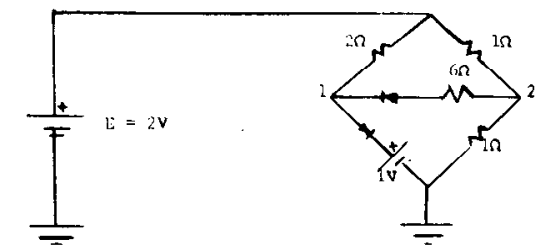

Fig. 5.

TABLE I

\begin{tabular}{c|c|c|c|c}
\hline$k$ & $\epsilon_{k}$ & $\begin{array}{c}\text { Number of } \\
\text { iterations }\end{array}$ & $\left(\mathrm{y}_{1}^{\mathrm{k}}, \mathrm{y}_{2}^{\mathrm{k}}\right)$ & $\left(\mathrm{v}_{1}^{\mathrm{k}}, \mathrm{v}_{2}{ }^{\mathrm{k}}\right)$ \\
\hline 0 & 1.000 & 3 & $(.10, .10)$ & $(1.8311, .99097)$ \\
1 & .1250 & 2 & $(.0181, .1025)$ & $(1.8016, .99999)$ \\
2 & .0156 & 2 & $(.0000, .0992)$ & $(1.8050,1.0000)$ \\
3 & .0002 & 1 & $(.0000, .0975)$ & $(1.3052,1.0000)$ \\
\hline
\end{tabular}

erated if successive values of $\epsilon_{k}$ are reduced as much as possible without inducing ill-conditioning. Also the initial values of the multipliers $y^{0}$ should be taken as close as possible to their final values $\bar{y}_{n}=\bar{i}_{n}+p_{n}$ (c.f. (14)).

As an example we give some computational results for a simple test problem.

\section{Test Problem}

Consider the network given in Fig. 5 containing two identical diodes.

Case 1: For this case the diodes have identical exponential characteristics of the form $i=0.1 \times 10^{-14}\left(e^{40 v}-\right.$ 1). The two equations for the voltages $v_{1}, v_{2}$ at the points 1 and 2 shown in the figure are as follows:

$$
\begin{gathered}
E-2 v_{2}-p\left[e^{q\left(13 v_{2}-v_{1}-6 E\right)}-1\right]=0 \\
1.5 E-2 v_{2}-0.5 v_{1}-p\left[e^{q\left(v_{1}-1\right)}-1\right]=0
\end{gathered}
$$

where

$$
p=0.1 \times 10^{-14}, \quad q=40, \quad E=2 .
$$

When the pure form of Newton's method was utilized to solve the equations above we obtained convergence in 12 iterations from the initial point $v_{1}=v_{2}=1$ to the final point $v_{1}=1.8052, v_{2}=1.0000$. However for the initial point $(3,0)$ Newton's method was progressing very slowly and failed to converge after 20 iterations while for the initial points $(0,4)$ and $(-2,6)$ overflow occurred immediately.

We now give in Table $I$ the results of the computation using our method. The table gives the parameter $\epsilon_{k}$ for the $k$ th network problem, the multiplier values $y_{1}^{k}, y_{2}^{k}\left(y_{1}^{k}, y_{2}^{k}\right.$ refer to the diodes in (15) and (16), respectively), the values of $v_{1}, v_{2}$ obtained, and the number of iterations of Newton's method. The initial point was $v_{1}=v_{2}=1$. Notice that the number of iterations is 8 (four less than for the ordinary Newton's method). For the same initial multipliers $y_{1}^{0}=y_{2}^{0}=0.1$ and other starting points $v_{1}, v_{2}$ we 
obtained similar results. For the initial point $v_{1}=3, v_{2}=0$ the number of iterations was 8 . For $v_{1}=0, v_{2}=4$ the number was 15 . For $v_{1}=-2, v_{2}=6$ the number was 11 . For $v_{1}=5, v_{2}=8$ the number was 12 . For $v_{1}=10, v_{2}=5$ the number was 15 . Thus there was no difficulty in solving the problem for widely varying initial conditions and furthermore the number of iterations required was not extremely sensitive to the initial values of $v_{1}$ and $v_{2}$. Similar results were obtained for $E=10$ and the same initial conditions although the number of iterations required was higher (around 30). This may be attributed to the fact that for $E=10$ the initial multiplier values $y_{1}^{0}=y_{2}^{0}$ $=0.1$ differ considerably from their final values.

Case 2: Here the two diodes are ideal. For $E=2, y_{1}^{0}=$ $y_{2}^{0}=0.1$ and a variety of initial values for $v_{1}, v_{2}$ the correct values for $v_{1}, v_{2}\left(v_{1}=v_{2}=1\right)$ was obtained within five significant digits in $30-35$ Newton iterations. Similar results were obtained for $E=10$.

\section{Monotone Networks-Relation to a Method of Multipliers}

We now provide convergence analysis of the algorithm proposed for the case of a monotone network as considered by Minty, Rockafellar, and others. In contrast with Section II we will not assume that the sequence $\left\{\epsilon_{k}\right\}$ utilized in the algorithm converges to zero. Instead we will assume that $\epsilon_{k}$ is kept constant although the proof of the result can be easily modified to cover the case where $\epsilon_{k}$ is not constant and is bounded above. We will also assume that the solution of the system of equations (1), (3), (4) is carried out exactly (i.e., $\gamma_{k}=0$ in (10)-(12)).

The case considered is when in addiition to the topological network equations

$$
\begin{aligned}
& A i=0 \\
& B v=0
\end{aligned}
$$

and the diode equations

$$
g_{n}\left(i_{n}, v_{n}\right)=i_{n}-p_{n}\left(e^{q_{n} v_{n}}-1\right)=0, \quad n=1, \cdots, s
$$

the currents and voltage differences across the nondiode arcs must lie on corresponding complete increasing curves as defined in Rockafellar [11]. These last relations are denoted by

$$
\left(i_{n}, v_{n}\right) \in \Gamma_{n}, \quad n=s+1, \cdots, r
$$

where $\Gamma_{n}$ are nonempty subsets of $R \times R$. We are seeking a solution to the system of relations (1), (2), (15).

Now by Theorem 1 of [11] a pair of vectors $(\bar{i}, \bar{v})$ solves (1), (2), (15) if and only if $\bar{i}$ solves a certain optimization problem (see [11]) and $\bar{v}$ solves the optimization problem

$$
\operatorname{minimize} \sum_{n=1}^{s}\left\{\frac{p_{n}}{q_{n}}\left(e^{q_{n} v_{n}}-1\right)-p_{n} v_{n}\right\}+\sum_{n=s+1}^{r} \varphi_{n}\left(v_{n}\right)
$$

subject to $B v=0$.
The functions $\varphi_{n}: R \rightarrow(-\infty,+\infty]$ above are constructed from the complete increasing curves $\Gamma_{n}$ of (15) as follows (see [11]). Define for all $v_{n} \in R$

$$
\Gamma_{n}\left(v_{n}\right)=\left\{i_{n} \mid\left(i_{n}, v_{n}\right) \in \Gamma_{n}\right\}
$$

and consider the interval

$$
I_{n}=\left\{v_{n} \mid \Gamma_{n}\left(v_{n}\right): \text { nonempty }\right\} \text {. }
$$

Then fix some $\tilde{v}_{n} \in I_{n}$ and define

$$
\begin{aligned}
& \varphi_{n}\left(v_{n}\right)=+\infty, \quad \text { if } v_{n} \notin I_{n} \\
& \varphi_{n}\left(v_{n}\right)=\int_{\tilde{v}_{n}}^{v_{n}} \gamma_{n}(w) d w, \quad \text { if } v_{n} \in I_{n}
\end{aligned}
$$

where for $w \in I_{n}, \gamma_{n}(w)$ is any scalar belonging to $\Gamma_{n}(w)$. As shown in [11] the functions $\varphi_{n}$ as defined above are convex and lower semicontinuous on $R$. Furthermore by [11, theorem 1'] a vector $\bar{v}$ solves the optimization problem (16) if and only if there exists a vector $i$ such that $(\bar{i}, \bar{v})$ solve the network problem (1), (2), (15). The algorithm that we propose will be studied in terms of the optimization problem (16) which is equivalent to the network problem under consideration in view of the discussion above.

Problem (16) is equivalent to the following problem

$\operatorname{minimize} \sum_{n=1}^{s}\left\{\frac{p_{n}}{q_{n}}\left[e^{q_{n}\left(v_{n}-u_{n}\right)}-1\right]-p_{n} v_{n}\right\}+\sum_{n=s+1}^{r} \varphi_{n}\left(v_{n}\right)$

subject to $B v=0, \quad u_{n} \leqslant 0 \quad, n=1, \cdots, s$

where we have introduced additional variables $u_{1}, \cdots, u_{s}$. $\Lambda$ vector $\bar{v}$ is an optimal solution of problem (16) if and only if $(\bar{v}, 0)$ is an optimal solution of problem (17). One may solve problem (17) numerically by using some constrained minimization method. In particular one may use a method from the class of multiplier algorithms. These algorithms have been proposed and analyzed recently (see [13], [14], [17], [18]). They are similar in spirit to classical penalty methods, but are much superior in terms of speed of convergence and reliability. A multiplier method such as those proposed in [14] and [18] when applied to problem (17) consists of sequential solution for $k=0,1, \cdots$, of the problem

$$
\begin{aligned}
\operatorname{minimize} & \sum_{n=1}^{s}\left\{\frac{p_{n}}{q_{n}}\left[e^{q_{n}\left(v_{n}-u_{n}\right)}-1\right]-p_{n} v_{n}+\epsilon_{k} p\left(\frac{u_{n}}{\epsilon_{k}}, y_{n}^{k}\right)\right\} \\
& +\sum_{n=s+1}^{r} \varphi_{n}\left(v_{n}\right)
\end{aligned}
$$

subject to $B v=0$

where $y_{n}^{k}$ are nonnegative scalars referred to as the multipliers and $\left\{\epsilon_{k}\right\}$ is a sequence of positive scalars referred to as the penalty parameter sequence. The real valued function $p$, referred to as a generalized penalty function, satisfies certain conditions (see [14], [18]) chief among 
which are continuity and convexity with respect to $u$ and

$$
\begin{aligned}
p(u, y) & >0, & \forall u>0, \\
p(u, y) & \leqslant 0, & \forall u \leqslant 0 \\
\lim _{u \rightarrow \infty} p(u, y) & =\infty & \\
\lim _{u \rightarrow-\infty} p(u, y) & >-\infty &
\end{aligned}
$$

for all $y \geqslant 0$. The most commonly used penalty function is the quadratic

$$
p(u, y)= \begin{cases}y u+\frac{1}{2} u^{2}, & u \geqslant-y \\ -\frac{1}{2} y^{2}, & u \leqslant-y\end{cases}
$$

however in our case it is essential to utilize the following exponential penalty function (see [14], p. 163):

$$
p(u, y)=y\left(e^{u}-1\right)
$$

for reasons that will become apparent shortly.

Multiplier methods can be operated similarly as penalty methods by taking $\epsilon_{k} \rightarrow 0$. The feature however which distinguishes them from penalty methods is that after each minimization of the form (18) the multipliers are updated by means of the iteration

$$
y_{n}^{k+1}=\frac{d}{d u} p\left(\frac{u_{n}^{k}}{\epsilon_{k}}, y_{n}^{k}\right), \quad n=1, \cdots, s
$$

where $u_{n}^{k} n=1, \cdots, s$ together with some vector $v^{k}=$ $\left(v_{1}^{k}, \cdots, v_{r}^{k}\right)$ solve problem (18). This iteration is aimed at choosing $y_{n}^{k+1}$ as close as possible to Lagrange multipliers corresponding to the incquality constraints in (17). The significant advantage of multiplier methods over penalty methods stems from the fact that iteration (20) can induce convergence of the method (i.e., $v^{k} \rightarrow \bar{v}, u_{n}^{k} \rightarrow \bar{u}_{n}$-a solution of (17)) even without taking $\epsilon_{k} \rightarrow 0$ as in penalty methods. Under convexity assumptions (see [14], [18]) it is merely sufficient to keep $\left\{\epsilon_{k}\right\}$ bounded above-for example, $\epsilon_{k} \equiv$ constant. In the absence of convexity it is necessary to have $\epsilon_{k} \leqslant \bar{\epsilon}$ for all $k$ sufficiently large where $\bar{\epsilon}>0$ is a scalar depending on the problem (see [13]). Thus the well known disadvantages of penalty methods associated with very small values of $\epsilon_{k}$ are alleviated. Furthermore the overall method requires significantly less computation time than a corresponding penalty method.

Let us now consider the multiplier method with the exponential penalty function (19) and $\epsilon_{k} \equiv \epsilon>0$ applied to problem (17). We introduce the vector $y=\left(y_{1}, \cdots, y_{s}\right)$ and we consider the Augmented Lagrangian defined by

$$
\begin{aligned}
L(v, u, y, \epsilon)=\sum_{n=1}^{s}\{ & \frac{p_{n}}{q_{n}}\left[e^{q_{n}\left(v_{n}-u_{n}\right)}-1\right]-p_{n} v_{n} \\
& \left.+\epsilon y_{n}\left(e^{u_{n} / \epsilon}-1\right)\right\}+\sum_{n=s+1}^{r} \varphi_{n}\left(v_{n}\right) .
\end{aligned}
$$

In the general iteration of the algorithm a multiplier vector $y^{k}=\left(y_{1}^{k}, \cdots, y_{s}^{k}\right)$ with positive coordinates is given and one obtains a pair of vectors $\left(v^{k}, u^{k}\right)$ which solve the problem

$$
\min _{B v=0} L\left(v, u, y^{k}, \epsilon\right)
$$

Subsequently the multiplier vector is updated by means of the iteration

$$
y_{n}^{k+1}=y_{n}^{k} e^{u_{n}^{k} / \epsilon}, \quad n=1, \cdots, s .
$$

The minimization cycle of (22) is repeated with $y^{k+1}$ replacing $y^{k}$. The initial multiplier vector $y^{0}$ is an arbitrary vector with positive coordinates.

Now the typical minimization cycle (22) can be carried out explicitly (i.e., in closed form) with respect to $u$. Indeed one may verify by straightforward calculation that

$$
\begin{aligned}
\min _{u_{n}}\left\{\frac{p_{n}}{q_{n}} e^{q_{n}\left(v_{n}-u_{n}\right)}+\epsilon y_{n} e^{u_{n} / \epsilon}\right\} \\
=\frac{1+q_{n} \epsilon}{q_{n}}\left(y_{n}\right)^{q_{n} \epsilon /\left(1+q_{n} \epsilon\right)}\left(p_{n}\right)^{1 /\left(1+q_{n} \epsilon\right)} e^{q_{n} v_{n} /\left(1+q_{n} \epsilon\right)}
\end{aligned}
$$

while the minimizing scalar $\tilde{u}_{n}$ above satisfies

$$
e^{\tilde{u}_{n} / \epsilon}=\left[\frac{p_{n}}{y_{n}} e^{q_{n} v_{n}}\right]^{1 /\left(1+q_{n} \epsilon\right)}
$$

In view of the relations above the minimization problem (22) can be written as

$$
\min _{B v=0} \tilde{L}\left(v, y^{k}, \epsilon\right)
$$

where the function $\tilde{L}$ is defined by

$$
\begin{aligned}
\tilde{L}\left(v, y^{k}, \epsilon\right)= & \sum_{n=1}^{s}\left\{\frac{1+q_{n} \epsilon}{q_{n}}\left(y_{n}^{k}\right)^{q_{n} \epsilon /\left(1+q_{n} \epsilon\right)}\right. \\
& \left.\cdot\left(p_{n}\right)^{1 /\left(1+q_{n} \epsilon\right)} e^{q_{n} v_{n} /\left(1+q_{n} \epsilon\right)}\right\} \\
& -\sum_{n=1}^{s}\left\{\frac{p_{n}}{q_{n}}+\epsilon y_{n}^{k}+p_{n} v_{n}\right\}+\sum_{n=s+1}^{r} \varphi_{n}\left(v_{n}\right) .
\end{aligned}
$$

Furthermore the multiplier iteration (23) can be written by using (24) as

$$
\begin{array}{r}
y_{n}^{k+1}=\left(y_{n}^{k}\right)^{q_{n} \varepsilon /\left(1+q_{n} \varepsilon\right)}\left(p_{n}\right)^{1 /\left(1+q_{n} \varepsilon\right)} e^{q_{n} v_{n} /\left(1+q_{n} \varepsilon\right)}, \\
n=1, \cdots, s .
\end{array}
$$

Thus even though the multiplier algorithm was defined in terms of the additional variables $u_{1}, \cdots, u_{s}$, the computations need not involve these variables and in its reduced form the algorithm consists of sequential minimizations of the form (25), (26) followed by multiplier updatings of the form (27). This important feature is present thanks to our particular choice of a penalty function with exponential form.

Now to relate the algorithm specified by (25)-(27) to the algorithm of Section II we need only observe that the minimization problem (25), (26) is, in view of the theorems of [11] mentioned earlier, equivalent to solving the 
system of relations

$$
\begin{array}{r}
A i=0, \quad B v=0, \quad\left(i_{n}, v_{n}\right) \in \Gamma_{n}, \quad n=s+1, \cdots, r \\
\tilde{g}_{n}\left(i_{n}, v_{n}, y_{n}^{k}, \epsilon\right)=i_{n}-\left(y_{n}^{k}\right)^{q_{n} \epsilon /\left(1+q_{n} \epsilon\right)}\left(p_{n}\right)^{1 /\left(1+q_{n} \epsilon\right)} e^{q_{n} v_{n} /\left(1+q_{n} \epsilon\right)} \\
+p_{n}=0, \quad n=1, \cdots, s .
\end{array}
$$

In conclusion the algorithm proposed in Section II (for the case where $\gamma_{k} \equiv 0, \epsilon_{k} \equiv \epsilon$ ) is equivalent to the method of multipliers specified by (21)-(23), or the method of multipliers specified by (25)-(27).

We now provide the following proposition the proof of which is similar to the proof of corresponding results in [14]. Actually in [14] the results obtained are weaker (a fact which prompted the authors of [14] to consider a slightly different class of algorithms for convex programming [17]-[19]). However for the special case under consideration the stronger convergence results given here may be obtained by exploiting.the fact that the sequences $\left\{y_{n}^{k}\right\}$ can be shown to be bounded away from zero.

\section{Proposition}

Let the assumptions of this section hold and assume that the system of relations (1), (2), (15) has a nonempty solution set $S^{*}$ which is bounded in $v$ (i.e., there exists a $\rho>0$ such that $\|\bar{v}\| \leqslant \rho$ for all $\left.(\bar{i}, \bar{v}) \in S^{*}\right) .{ }^{1}$

a) For every $y^{k}=\left(y_{1}^{k}, \cdots, y_{s}^{k}\right)$ with $y_{n}^{k}>0, n=1, \cdots, s$, and $\epsilon>0$ the system of relations (28), (29) has a nonempty solution set which is bounded in $v$.

b) Let $\left\{v^{k}, y^{k}\right\}$ be a sequence generated by the algorithm which consists of repetitive solution of the system (28), (29) with the multiplier vectors $y^{k}$ updated by (27). Then the sequence $\left\{v^{k}, y^{k}\right\}$ is bounded. Furthermore if $(\bar{v}, \bar{y})$ is any one of its limit points, then there exists a vector $\bar{i} \in R^{r}$ such that the pair $(\bar{i}, \bar{v})$ is a solution of the system (1), (2), (15) and $\bar{y}$ satisfies

$$
\bar{y}_{n}=\bar{i}_{n}+p_{n}=p_{n} e^{q_{n} \bar{v}_{n}}, \quad n=1, \cdots, s .
$$

Proof: The proof relies heavily on the theory of convex functions and convex programming as presented in Rockafellar's text [22].

a) Using the notation (21) we have for any $y^{k}, \tilde{y}$ with $y_{n}^{k} \geqslant 0, \tilde{y}_{n} \geqslant 0, n=1, \cdots, s$

$$
\begin{aligned}
L\left(v, u, y^{k}, \epsilon\right)= & \sum_{n=1}^{s}\left\{\frac{p_{n}}{q_{n}}\left[e^{q_{n}\left(v_{n}-u_{n}\right)}-1\right]-p_{n} v_{n}\right. \\
& \left.+\epsilon y_{n}^{k}\left(e^{u_{n} / \epsilon}-1\right)\right\}+\sum_{n=s+1}^{r} \varphi_{n}\left(v_{n}\right) \\
\geqslant & \sum_{n=1}^{s}\left\{\frac{p_{n}}{q_{n}}\left[e^{q_{n}\left(v_{n}-u_{n}\right)}-1\right]-p_{n} v_{n}+\tilde{y}_{n} u_{n}\right\} \\
& +\sum_{n=s+1}^{r} \varphi_{n}\left(v_{n}\right)+\sum_{n=1}^{s} \inf _{t}\left\{\epsilon y_{n}^{k}\left(e^{t / \epsilon}-1\right)-\tilde{y}_{n} t\right\} .
\end{aligned}
$$

\footnotetext{
${ }^{1}$ Conditions for this to occur are given in [20], [21].
}

Notice that the last term on the right above is real valued and bounded for any fixed $\tilde{y}$ and a bounded sequence $\left\{y^{k}\right\}$. We will show that problem (22) has a nonempty and compact optimal solution set. To this end we must show that the function $L\left(v, u, y^{k}, \epsilon\right)$ has no direction of recession $(\tilde{v}, \tilde{u})$ with $B \tilde{v}=0$ (see $[22$, p. 69] for the definition of a direction of recession; also [22, cor. 8.7.1, th. 27.1d). Let $(\tilde{v}, \tilde{u})$ be such that $(\tilde{v}, \tilde{u}) \neq(0,0)$ and $B \tilde{v}=0$. Our assumption that the system of relations (1), (2), (15) has a nonempty solution set bounded in $v$ implies that problem (17) has a nonempty and compact solution set. Hence there are two possibilities. Either $(\tilde{v}, \tilde{u})$ satisfies $\tilde{u}_{n} \leqslant 0$, $n=1, \cdots, s$ and $(\tilde{v}, \tilde{u})$ is a direction of recession of the objective function of problem (17), given below for convenience

$$
\sum_{n=1}^{s}\left\{\frac{p_{n}}{q_{n}}\left[e^{q_{n}\left(v_{n}-u_{n}\right)}-1\right]-p_{n} v_{n}\right\}+\sum_{n=s+1}^{r} \varphi_{n}\left(v_{n}\right)
$$

or else $\tilde{u}_{\tilde{n}}>0$ for some $\tilde{n} \in\{1, \cdots, s\}$. If $\tilde{u}_{n} \leqslant 0, n=1, \cdots, s$ then by taking $\tilde{y}=0$ in (31) the right side is the same as the objective function of problem (17) plus a constant and it follows that $(\tilde{v}, \tilde{u})$ cannot be a direction of recession of $L\left(v, u, y^{k}, \epsilon\right)$. If $\tilde{u}_{\tilde{n}}>0$ for some $\tilde{n} \in\{1, \cdots, s\}$ then take $\tilde{y}_{n}=0$ for $n \neq \tilde{n}$ in (31) and take $\tilde{y}_{\tilde{n}}$ sufficiently large to guarantee that $(\tilde{v}, \tilde{u})$ is not a direction of recession of the resulting function on the right. Since it is possible to choose $\tilde{y}_{\tilde{n}}$ in this way (see [22, th. 8.8, cor. 8.7.1, th. 9.3]) it follows that $(\tilde{v}, \tilde{u})$ is not a direction a recession of $L\left(v, u, y^{k}, \epsilon\right)$. Hence problem (22) has a nonempty and compact solution set and by [11, th. 1'] the set of relations (28), (29) has a nonempty solution set bounded in $v$.

b) The proof of this part will be developed through several lemmas. Consider the ordinary dual functional $d: R^{s} \rightarrow[-\infty, \infty)$ of problem (17) defined for each $y=$ $\left(y_{1}, \cdots, y_{s}\right)$ by

$$
d(y)=\left\{\begin{array}{c}
\inf _{B v=0}\left[\sum_{n=1}^{s}\left\{\frac{p_{n}}{q_{n}}\left[e^{q_{n}\left(v_{n}-u_{n}\right)}-1\right]-p_{n} v_{n}+y_{n} u_{n}\right\}\right. \\
\left.+\sum_{n=s+1}^{r} \varphi_{n}\left(v_{n}\right)\right], \quad \text { if } y_{n} \geqslant 0, n=1, \cdots, s \\
-\infty, \quad \text { otherwise }
\end{array} .\right.
$$

A vector $\bar{y}$ is a maximizing point of $d$ if and only if it is a Lagrange multiplier vector of problem $(17)([22,828])$ in the sense that $\bar{y}_{n} \geqslant 0, n=1, \cdots, s$ and

$$
\begin{aligned}
\min _{B v=0}\left[\sum_{n=1}^{s}\right. & \left\{\frac{p_{n}}{q_{n}}\left[e^{q_{n}\left(v_{n}-u_{n}\right)}-1\right]-p_{n} v_{n}+\bar{y}_{n} u_{n}\right\} \\
& \left.+\sum_{n=s+1}^{r} \varphi_{n}\left(v_{n}\right)\right] \\
= & \sum_{n=1}^{s}\left\{\frac{p_{n}}{q_{n}}\left(e^{q_{n} \bar{v}_{n}}-1\right)-p_{n} \bar{v}_{n}\right\}+\sum_{n=s+1}^{r} \varphi_{n}\left(\bar{v}_{n}\right)
\end{aligned}
$$

where $\bar{v}$ is an optimal solution of problem (16) or equivalently $(\bar{v}, 0)$ is an optimal solution of problem (17). Since 
$(\bar{v}, 0)$ attains the minimum in the left side of $(34)$ we obtain by differentiation with respect to $u_{n}$

$$
-p_{n} e^{q_{n} \bar{v}_{n}}+\bar{y}_{n}=0
$$

Hence

$$
\bar{y}_{n}=p_{n} e^{q_{n} \bar{v}_{n}}=\bar{i}_{n}+p_{n}, \quad n=1, \cdots, s
$$

where $\bar{i}$ together with $\bar{v}$ are a solution to the network equations (1), (2), (15). Since the set of all such $\bar{v}$ is nonempty and compact by our assumption the set of all $\bar{y}$ of (35) is also nonempty and compact. We state our conclusion as a lemma.

\section{Lemma 1}

A vector $\bar{y}=\left(\bar{y}_{1}, \cdots, \bar{y}_{s}\right)$ is a maximizing point of the dual functional $d$ of (33) if and only if

$$
\bar{y}_{n}=p_{n} e^{q_{n} \bar{v}_{n}}=\bar{i}_{n}+p_{n}, \quad n=1, \cdots, s
$$

where $(\bar{i}, \bar{v})$ is some solution of the network equations (1), (2), (15). The set of all these maximizing points is nonempty and compact.

Consider now the function $d_{\epsilon}: R^{s} \rightarrow[-\infty, \infty)$ defined for each $y=\left(y_{1}, \cdots, y_{s}\right)$ and $\epsilon>0$ by

$$
d_{\epsilon}(y)=\left\{\begin{array}{l}
\inf _{B v=0}\left[\sum_{n=1}^{s}\left\{\frac{p_{n}}{q_{n}}\left[e^{q_{n}\left(v_{n}-u_{n}\right)}-1\right]-p_{n} v_{n}+\epsilon y_{n}\left(e^{u_{n} / e}-1\right)\right\}+\sum_{n=s+1}^{r} \varphi_{n}\left(v_{n}\right)\right] . \\
=\inf _{B v=0} L(v, u, y, \epsilon), \quad \text { if } y_{n} \geqslant 0, \quad n=1, \cdots, s \\
-\infty, \quad \text { otherwise }
\end{array}\right.
$$

each $k \geqslant 1$ :

$$
\begin{gathered}
-\infty<d\left(y^{k}\right)<d_{\epsilon}\left(y^{k}\right)<d\left(y^{k+1}\right), \quad \text { if } y^{k} \neq y^{k+1} \\
d\left(y^{k}\right)=d_{\epsilon}\left(y^{k}\right)=\max _{y} d(y), \quad \text { if } y^{k}=y^{k+1} .
\end{gathered}
$$

Furthermore $\left\{v^{k}, u^{k}, y^{k}\right\}$ is a bounded sequence.

Proof: Consider the sequences $\left\{u_{n}^{k}\right\}, n=1, \cdots, s$ defined by $y_{n}^{k+1}=y_{n}^{k} e^{u_{n}^{k} / \epsilon}$ (c.f. (23)). Then $\left(v^{k}, u^{k}\right)$ solve for each $k$ the problem

$$
\min _{B v=0} L\left(v, u, y^{k}, \epsilon\right) .
$$

If $y^{k} \neq y^{k+1}$ then $u_{\tilde{n}}^{k} \neq 0$ for some $\tilde{n} \in\{1, \cdots, s\}$ and a simple calculation shows that

$$
u_{n}^{k}<\epsilon\left(e^{u_{n}^{k} / \epsilon}-1\right)<u_{n}^{k} e^{u_{n}^{k} / \epsilon}
$$

while for all $n$ we have

$$
u_{n}^{k} \leqslant \epsilon\left(e^{u_{n}^{k} / \epsilon}-1\right) \leqslant u_{n}^{k} e^{u_{n}^{k} / \epsilon}, \quad n=1, \cdots, s .
$$

Now from (23), (33), (36)-(38) and the fact $y_{n}^{k}>0, n=$
This is the dual functional associated with the problem

$$
\operatorname{minimize} \sum_{n=1}^{s}\left\{\frac{p_{n}}{q_{n}}\left[e^{q_{n}\left(v_{n}-u_{n}\right)}-1\right]-p_{n} v_{n}\right\}+\sum_{n=s+1}^{r} \varphi_{n}\left(v_{n}\right)
$$$$
\text { subject to } B v=0, \quad \epsilon\left(e^{u_{n} / \epsilon}-1\right) \leqslant 0, \quad n=1, \cdots, s \text {. }
$$

The problem above has the same optimal solutions as problem (17) and by repeating the procedure given earlier we obtain the following.

\section{Lemma 2}

The set of maximizing points of the dual functional $d_{\epsilon}$ of (36) is identical to the set of maximizing points of the dual functional $d$ of (33).

We now show the following crucial lemma which demonstrates that our algorithm may be viewed as an ascent method for maximizing both $d^{\prime}$ and $d_{\epsilon}$.

\section{Lemma 3}

For a sequence $\left\{v^{k}, u^{k}, y^{k}\right\}$ generated by the algorithm where $u^{k}$ is defined by (23) the following hold true for
$1, \cdots, s$ we obtain

$$
\begin{aligned}
d\left(y^{k}\right) \leqslant & \sum_{n=1}^{s}\left\{\frac{p_{n}}{q_{n}}\left[e^{q_{n}\left(v_{n}^{k}-u_{n}^{k}\right)}-1\right]-p_{n} v_{n}^{k}+y_{n}^{k} u_{n}^{k}\right\} \\
& +\sum_{n=s+1}^{r} \varphi_{n}\left(v_{n}^{k}\right) \\
< & \sum_{n=1}^{s}\left\{\frac{p_{n}}{q_{n}}\left[e^{q_{n}\left(v_{n}^{k}-u_{n}^{k}\right)}-1\right]-p_{n} v_{n}^{k}+\epsilon y_{n}^{k}\left(e^{u_{n}^{k} / \epsilon}-1\right)\right\} \\
& +\sum_{n=s+1}^{r} \varphi_{n}\left(v_{n}^{k}\right)=d_{\epsilon}\left(y^{k}\right) \\
< & \sum_{n=1}^{s}\left\{\frac{p_{n}}{q_{n}}\left[e^{q_{n}\left(v_{n}^{k}-u_{n}^{k}\right)}-1\right]-p_{n} v_{n}^{k}+y_{n}^{k+1} u_{n}^{k}\right\} \\
& +\sum_{n=s+1}^{r} \varphi_{n}\left(v_{n}^{k}\right)=d\left(y^{k+1}\right) .
\end{aligned}
$$

The last equality follows from the fact that $\left(v^{k}, u^{k}\right)$ satisfy the necessary conditions for minimizing over all $(v, u)$ with $B v=0$ the function

$$
\sum_{n=1}^{s}\left\{\frac{p_{n}}{q_{n}}\left[e^{q_{n}\left(v_{n}-u_{n}\right)}-1\right]-p_{n} v_{n}+y_{n}^{k+1} u_{n}\right\}+\sum_{n=s+1}^{r} \varphi_{n}\left(v_{n}\right) .
$$


This can be seen from the fact that $\left(v^{k}, u^{k}\right)$ solve problem (22) and from the updating formula (23). To show $d\left(y^{k}\right)$ $>-\infty$ for all $k>1$ we note that we have $d_{\mathrm{e}}\left(y^{k}\right)>-\infty$ for all $k \geqslant 0$ since the infimum in (36) is attained. It follows that $d\left(y^{k+1}\right) \geqslant d_{\epsilon}\left(y^{k}\right)>-\infty$ for all $k \geqslant 0$. The preceding proof also shows that $d\left(y^{k}\right) \leqslant d_{\mathrm{e}}\left(y^{k+1}\right) \leqslant d\left(y^{k+1}\right)$ for all $k$ and hence if $y^{k}=y^{k+1}$ equality holds throughout. Also if $y^{k}=y^{k+1}$ it follows that $u_{n}^{k}=0, n=1, \cdots, s$ and $\left(v^{k}, 0\right)$ is an optimal solution of problem (17). Hence $y^{k+1}$ is a Lagrange multiplier vector for problem (17) and $d\left(y^{k+1}\right)$ $=\max _{y} d(y)$.

Finally to show that $\left\{v^{k}, u^{k}, y^{k}\right\}$ is a bounded sequence observe that

$$
y^{k} \in\left\{y \mid d_{\epsilon}(y) \geqslant d_{\epsilon}\left(y^{0}\right)\right\}, \quad k=0,1, \cdots .
$$

The set of maximizing points of $d_{\epsilon}$ is nonempty and compact by Lemmas 1 and 2 . Hence all its level sets are compact and in particular the set in (39) is compact ([22, cor. 8.7.1]). Hence $\left\{y^{k}\right\}$ is bounded. To show that $\left\{v^{k}, u^{k}\right\}$ is bounded consider (31). Take $\tilde{y}_{n}, n=1, \cdots, s$ sufficiently large so that the function

$$
\begin{aligned}
\hat{L}(v, u, \tilde{y}, \epsilon)=\sum_{n=1}^{s}\left\{\frac{p_{n}}{q_{n}}\left[e^{q_{n}\left(v_{n}-u_{n}\right)}-1\right]-p_{n} v_{n}\right. & \left.+\tilde{y}_{n} u_{n}\right\} \\
& +\sum_{n=s+1}^{r} \varphi_{n}\left(v_{n}\right)
\end{aligned}
$$

has no directions of recession and hence its level sets are compact. Let $\delta \in R$ be such that

$$
\delta \leqslant \sum_{n=1}^{s} \inf _{t}\left\{\boldsymbol{\epsilon} y_{n}^{k}\left(e^{t / \epsilon}-1\right)-\tilde{y}_{n} t\right\}, \quad k=0,1, \cdots .
$$

Such a $\delta$ exists by boundedness of $\left\{y^{k}\right\}$. Then (31) yields

$$
\begin{aligned}
\max _{y} d(y) & \geqslant d\left(y^{k+1}\right) \geqslant d_{\epsilon}\left(y^{k}\right)=L\left(v^{k}, u^{k}, y^{k}, \epsilon\right) \\
& \geqslant \hat{L}\left(v^{k}, u^{k}, \tilde{y}, \epsilon\right)+\delta .
\end{aligned}
$$

Hence $\left(v^{k}, u^{k}\right)$ belongs to the compact set

$$
\left\{(v, u) \mid \hat{L}(v, u, \tilde{y}, \epsilon) \leqslant \max _{y} d(y)-\delta\right\}
$$

and $\left\{v^{k}, u^{k}\right\}$ is bounded.

Q.E.D.

We are now ready to prove the last part of the proposition. Let $\left\{v^{k}, u^{k}, y^{k}\right\}$ be a sequence generated by sequential solution of the problem

$$
\min _{B v=0} L\left(v, u, y^{k}, \epsilon\right)
$$

with $y^{k}$ updated by (27). This sequence is bounded by Lemma 3 . We have from the necessary conditions for optimality for problem (40)

$$
-p_{n} e^{q_{n}\left(v_{n}^{k}-u_{n}^{k}\right)}+y_{n}^{k} e^{u_{n}^{k} / \epsilon}=0 .
$$

If $(\bar{v}, \bar{u}, \bar{y})$ is any limit point of $\left\{v^{k}, u^{k}, y^{k}\right\}$ we have from
(41)

$$
\bar{y}_{n}=p_{n} e^{q_{n}\left(\bar{v}_{n}-\bar{u}_{n}\right)-\left(\bar{u}_{n} / \epsilon\right)} \neq 0, \quad n=1, \cdots, s .
$$

By the definitions (33) and (36) of $d$ and $d_{\mathrm{c}}$ we have

$$
\begin{aligned}
d_{\epsilon}\left(y^{k}\right)= & \min _{B v=0} L\left(v, u, y^{k}, \epsilon\right)=\sum_{n=1}^{s}\left\{\frac{p_{n}}{q_{n}}\left[e^{q_{n}\left(v_{n}^{k}-u_{n}^{k}\right)}-1\right]\right. \\
& \left.-p_{n} v_{n}^{k}+\epsilon y_{n}^{k}\left(e^{u_{n}^{k} / \epsilon}-1\right)\right\}+\sum_{n=s+1}^{r} \varphi_{n}\left(v_{n}^{k}\right) \\
d\left(y^{k}\right) \leqslant & \sum_{n=1}^{s}\left\{\frac{p_{n}}{q_{n}}\left[e^{q_{n}\left(v_{n}^{k}-u_{n}^{k}\right)}-1\right]-p_{n} v_{n}^{k}+y_{n}^{k} u_{n}^{k}\right\} \\
& +\sum_{n=s+1}^{r} \varphi_{n}\left(v_{n}^{k}\right) .
\end{aligned}
$$

From the relations above and (38) we obtain

$$
d_{\epsilon}\left(y^{k}\right)-d\left(y^{k}\right) \geqslant y_{n}^{k}\left[\epsilon\left(e^{u_{n}^{k} / \epsilon}-1\right)-u_{n}^{k}\right] \geqslant 0
$$

we have $\left\{d_{\epsilon}\left(y^{k}\right)-d\left(y^{k}\right)\right\} \rightarrow 0$ by using the monotonicity and boundedness of $\left\{d\left(y^{k}\right)\right\}$ (c.f. Lemma 3). Hence (43) and (42) yield that

$$
\epsilon\left(e^{\bar{u}_{n} / \epsilon}-1\right)-\bar{u}_{n}=0
$$

from which

$$
\bar{u}_{n}=0, \quad n=1, \cdots, s .
$$

From (42) and (44) we obtain

$$
\bar{y}_{n}=p_{n} e^{q_{n} \bar{v}_{n}}, \quad n=1, \cdots, s .
$$

Also by lower semicontinuity of $\varphi_{n}$ and (44) we obtain

$$
\begin{aligned}
\max _{y} d(y) \geqslant \lim _{k \rightarrow \infty} L\left(v^{k}, u^{k}, y^{k}, \epsilon\right) \\
\geqslant \sum_{n=1}^{s}\left\{\frac{p_{n}}{q_{n}}\left[e^{q_{n}\left(\bar{v}_{n}-\bar{u}_{n}\right)}-1\right]-p_{n} \bar{v}_{n}+\epsilon \bar{y}_{n}\left(e^{\bar{u}_{n} / \epsilon}-1\right)\right\} \\
\quad+\sum_{n=s+1}^{r} \varphi_{n}\left(\bar{v}_{n}\right) \\
=\sum_{n=1}^{s}\left\{\frac{p_{n}}{q_{n}}\left(e^{q_{n} \bar{v}_{n}}-1\right)-p_{n} \bar{v}_{n}\right\}+\sum_{n=s+1}^{r} \varphi_{n}\left(\bar{v}_{n}\right) .
\end{aligned}
$$

Since $(\bar{v}, \bar{u})=(\bar{v}, 0)$ is also a feasible vector for problem (17) it follows that $(\bar{v}, 0)$ is an optimal solution for problem (17). Hence $\bar{v}$ is an optimal solution for problem (16) and by $\left[11\right.$, th. $\left.1^{\prime}\right]$ there exists a vector $i$ such that $(\bar{v}, i)$ solve the set of relations (1), (2), (15). Using also (45) it follows that $\bar{i}_{n}, \bar{v}_{n}$, and $\bar{y}_{n}, n=1, \cdots, s$ satisfy the relation stated in the proposition.

Q.E.D.

We note finally that the approach of this paper could. be used to construct algorithms for solution of network problems involving "ill conditioned" arc characteristics other than exponential such as, for example, discontinuous or nondifferentiable characteristics. The general idea is to convert the problem to an equivalent optimization 
problem and subsequently approximate this problem by utilizing an appropriate multiplier method (i.c., an appropriate penalty function which "matches" the problem) from the wide class of methods available [14], [17]-[19].

\section{Conclusions}

The algorithm presented in this paper represents a simple way to overcome the numerical difficulties associated with steeply rising diode characteristics in resistive network problems. As such it can be viewed as a method for increasing the reliability of network analysis algorithms. Since the nature of the method is such that the basic network structure of the problem is not affected, the procedure proposed can be very easily incorporated within existing programs.

\section{REFERENCES}

[1] N. Balabanian and T. A. Bickart, Electrical Network Theory. New York: Wiley, 1969

[2] J. M. Ortega and W. C. Rheinboldt, Iterative Solution of Nonlinear Equations in Several Variables. New York: Academic Press, 1970, p. 421.

[3] D. A. Calahan, Computer-Aided Network Design. New York: McGraw-Hill, 1972.

[4] F. H. Branin, G. R. Hogsett, R. L. Lunde, and L. E. Kugel, "ECAP II-a new electronic circuit analysis program," IEEE $J$. Solid-State Circuits, vol. SC-6, pp. 146-166, 1971

[5] G. C. Brown, "DC analysis of nonlinear networks," Electron. Lett., vol. 5, pp. 374-375, 1969.

[6] L. Nagel and R. Rohrer, "Computer analysis of nonlinear circuits, excluding radiation (CANCER)," IEEE J. Solid-State Circuits, vol. SC-6, pp. 166-182, 1971.

[7] W. J. Calla, and W. G. Howard, "Bias-3, a program for the nonlinear dc analysis of bipolar transistor circuits," IEEE $J$. of Solid State Circuits, vol. SC-6, pp. 14-19, 1971.

[8] D. F. Davidenko, "On a New Method of Numerical Solution of Systems of Nonlinear Equations," Dokl. Akad., Nauk SSSR, vol. 88, pp. 601-602, 1953.

[9] I. A. Cermak, "DC solution of nonlinear state space equations in circuit analysis," IEEE Trans. Circuit Theory, vol. CT-18, pp. 312-314, 1971

[10] G. J. Minty, "Monotone networks," Proc. Royal Soc. London, Series A 257, pp. 194-212, 1960.

[11] R. T. Rockafellar, "Convex programming and systems of elementary monotonic relations," J. Math. Analysis and Applications, vol. 19, pp. 543-564, 1967.

[12] - "Optimization in Networks," Lecture Notes, University of Grenoble, Grenoble, France, 1974.

[13] D. P. Bertsekas, "Multiplier methods: A survey," Automatica, vol. 12, pp. 133-145, 1976.

[14] B. W. Kort and D. P. Bertsekas, "A new penalty function method for constrained minimization," in Proc. 1972 IEEE Conf. on Decision and Control, New Orleans, LA, pp. 162-166.

[15] D. P. Bertsekas, "A general method for approximation based on the method of multipliers," in Proc. Thirteenth Annual Allerton Conf. on Circuit and System Theory, Allerton Park, IL, Oct. 1975.

[16] "Nondifferentiable optimization via approximation" in Mathematical Programming Study 3, M. Balinski and P. Wolfe, Eds. Amsterdam, The Netherlands: North Holland, 1975, pp. $1-25$.

[17] B. W. Kort and D. P. Bertsekas, "Multiplier methods for convex programming," in Proc. 1973 IEEE Conf. on Decision and Control, San Diego, CA, pp. 428-432.

[18] - "Combined primal-dual and penalty methods for constrained minimization," SIAM J. on Control, vol. 14, pp. 268-294, 1976.

[19] B. W. Kort, "Combined primal-dual and penalty function algorithms for nonlinear programming," Ph.D. dissertation, Stanford University, Palo Alto, CA, 1975.

[20] M. Iri, Network Flow, Transportation and Scheduling Theory and Algorithm. New York: Academic Press, 1969

[21] C. A. Desoer, and F. F. Wu, "Nonlinear monotone networks," SIAM J. Appl. Math., vol. 26, pp. 315-333, 1974.

[22] R. T. Rockafellar, Convex Analysis. Princeton, NJ: Princeton Univ. Press, 1970.

[23] L. Chua and S. Wang, "A new approach to overcome the overflow problem in computer-aided analysis of nonlinear resistive circuits," Inter. J. Circuit Theory and Appl., vol. 3, pp. 261-284, 1975.

[24] D. P. Bertsekas, "Minimax methods based on approximation," Proc. of Johns Hopkins Conf. on Information Sciences and Systems, Baltimore, MD, Mar. 1976.

[25] - "Approximation procedures based on the method of multipliers," J. Optimization Theory and Applications, to be published.

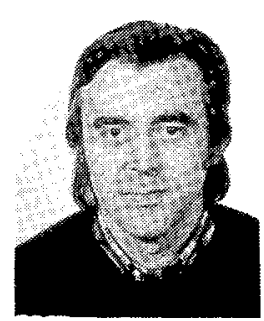

Dimitri P. Bertsekas was born in $\Lambda$ thens, Greece, in 1942. He received the Mechanical and Electrical Engineering Diploma from the National Technical University of Athens, Athens, Greece, in 1965, the M.S.E.E. degree from George Washington University, Washington, D. C., in 1969, and the Ph.D. degree in system science from the Massachusetts Institute of Technology, Cambridge, in 1971.

From 1966 to 1967 he performed research at the National Technical University of Athens, and from 1967 to 1969 he was with the U. S. Army Research Laboratories, Fort Belvoir, VA. In the summer of 1971 he worked at Systems Control, Inc., Palo Alto, CA. From September 1971 until December 1973 he was on the faculty of the Engineering-Fconomic Systems Department of Stanford University, Stanford, CA. In January 1974 he joined the Coordinated Science Laboratory and the Department of Electrical Engineering at the University of Illinois, Urbana, where he is currently an Associate Professor. He has done research in estimation and control of uncertain systems, dynamic programming, optimization theory, nonlinear programming algorithms, and economic analysis. He has authored or coauthored over forty research papers in these areas and he is the author of the graduate text Dynamic Programming and Stochastic Control (New York: Academic Press, 1976). 